\title{
Desigualdades de gênero por área de conhecimento na ciência brasileira: panorama das bolsistas $\mathrm{PQ} / \mathrm{CNPq}$
}

\author{
Gender inequalities by field of knowledge in Brazilian science: an \\ overview of the $P Q / C N P q$ female researchers
}

Rocelly Cunha', Magda Dimenstein ${ }^{\mathbf{1}}$, Candida Dantas ${ }^{\mathbf{1}}$

DOI: 10.1590/0103-11042021E107

\begin{abstract}
RESUMO O número de mulheres pesquisadoras tem crescido mundialmente. No entanto, as desigualdades de gênero persistem em quatro aspectos: as mulheres ainda representam parcela minoritária na ciência mundial; concentram-se em determinadas áreas de conhecimento; predominam nos níveis iniciais da carreira e são sub-representadas em posições deliberativas da política científica e tecnológica. No Conselho Nacional de Desenvolvimento Científico e Tecnológico (CNPq), apesar do aumento de mulheres bolsistas de Produtividade em Pesquisa (PQ) nas últimas décadas, as assimetrias permanecem. Este estudo visou discutir as assimetrias de gênero e raça nas diferentes áreas do conhecimento, em particular na psicologia, tomando como analisador a distribuição de bolsas (PQ) do CNPq. Utilizaram-se dados disponibilizados pelo CNPq e coletados por meio de SurveyMonkey. As desigualdades de gênero na ciência persistem no sistema científico brasileiro: as mulheres são minoria entre os bolsistas $\mathrm{PQ} / \mathrm{CNPq}$, concentram-se em guetos disciplinares e enfrentam dificuldades tanto para acessar o sistema PQ quanto para alcançar as modalidades de bolsa de maior prestígio científico. Na psicologia, apesar da presença em todas as modalidades de bolsa, ocupam proporcionalmente menos posições no topo da carreira. Ademais, há invisibilidade de mulheres negras e indígenas, a qual tem suas raízes no projeto moderno colonial.
\end{abstract}

PALAVRAS-CHAVE Ciência. Identidade de gênero. Grupos de populações continentais. Psicologia.

1 Universidade Federal do Rio Grande do Norte (UFRN) - Natal (RN), Brasil. rocellycunha@gmail.com
ABSTRACT The number of women researchers has grown worldwide. However, gender inequalities persist in four aspects: women still represent a minority share in world science; they are concentrated in certain fields of knowledge; they predominate in early career levels; and they are underrepresented in deliberative positions of science and technology policies. At the National Council for Scientific and Technological Development (CNPq), despite the increase in women Research Productivity Scholars $(P Q)$ in recent decades, the asymmetries remain. This study aims to discuss the asymmetries of gender and race in different fields of knowledge, particularly in Psychology, taking as an analyzer the distribution of grants (PQ) by the CNPq. Data made available by the CNPq and collected through SurveyMonkey were used. Gender inequalities in science persist in the Brazilian scientific system: women are a minority among the $P Q / C N P q$ fellows, they are concentrated in disciplinary ghettos and face difficulties both to access the PQ system, and to reach the most prestigious scientific fellowships. In Psychology, despite their presence in all the scholarship modalities, they occupy proportionally fewer positions at the top of the career. In addition, there is the invisibility of black and indigenous women, which has its roots in the modern colonial project.

KEYWORDS Science. Gender identity. Continental population groups. Psychology. 


\section{Introdução}

Este estudo visa contribuir com o debate acerca da participação das mulheres na produção de conhecimento e das profundas desigualdades de gênero existentes na ciência ${ }^{\mathbf{1}-4}$. Pretende, a partir da apresentação do panorama geral da posição das mulheres na ciência brasileira, discutir as assimetrias de gênero e raça nas diferentes áreas do conhecimento, em particular, na psicologia, tomando como analisador a distribuição de bolsas de Produtividade em Pesquisa (PQ) do Conselho Nacional de Desenvolvimento Científico e Tecnológico (CNPq).

A desigualdade de gênero na ciência é uma realidade em diferentes regiões e países. De acordo com a Organização das Nações Unidas para a Educação, a Ciência e a Cultura (Unesco) ${ }^{5}$, as mulheres representam atualmente apenas $30 \%$ dos pesquisadores no mundo. Estudos realizados por Mason e Ekman ${ }^{6}$ nos Estados Unidos; por Alonso, Diz e Lois ${ }^{7}$ na Espanha; por Bargillole e Goode ${ }^{8}$ no Reino Unido, bem como pela Elsevier9,10, revelaram a existência de padrões desiguais estruturados em pelo menos quatro aspectos: a) mulheres ainda representam parcela minoritária na ciência mundial; b) concentram-se em determinadas áreas de conhecimento; c) predominam nos níveis iniciais da carreira e, d) estão sub-representadas em posições deliberativas da política científica e tecnológica.

Estudos desenvolvidos no Brasil revelam o mesmo cenário. Barros e Mourão ${ }^{3}$; Valentova, Otta, Silva et al. ${ }^{\mathbf{4}}$; Tabak ${ }^{11}$; Leta ${ }^{12}$; Vasconcelos e Brisolla ${ }^{13}$; e Velho e Léon ${ }^{\mathbf{1 4}}$ constataram desigualdade entre mulheres e homens em termos de segregação horizontal e vertical, isto é, as mulheres são maioria em áreas do conhecimento relacionadas com as profissões socialmente identificadas como femininas e há uma proporção expressiva de mulheres em posições mais baixas na hierarquia da Ciência, Tecnologia e Inovação (CT\&I).

Apesar das mudanças recentes em relação à ampliação da participação das mulheres nas posições de destaque em CT\&I - a exemplo da bolsa PQ do CNPq -, ainda há muita assimetria em várias áreas do conhecimento, em especial, naquelas historicamente dominadas pelos homens. Ao analisar tais desigualdades, levando em conta o perfil étnico-racial, o cenário torna-se mais devastador. No Brasil, estima-se que apenas $10,4 \%$ das mulheres negras têm acesso ao ensino superior; e menos $3 \%$, à atividade de ensino e pesquisa nas instituições acadêmicas ${ }^{15}$. Esse quadro está intimamente relacionado com as sobreposições entre gênero, raça e classe, resultantes das marcas do sistema moderno colonial ${ }^{16,17}$ na ciência nacional que pode ser identificado na medida em que mulheres negras e pobres têm menos acesso, enfrentam mais obstáculos e competição do que mulheres brancas e de segmentos sociais privilegiados em relação à educação superior e à carreira acadêmica.

Na psicologia, o cenário de desequilíbrio étnico-racial tem a mesma configuração: entre as pós-graduandas em psicologia do País, menos de $12 \%$ são mulheres negras, e menos de $1 \%$ é mulher indígena ${ }^{18}$. Essa situação relaciona-se com o histórico da área, majoritariamente ocupada pela população branca ${ }^{19} \mathrm{e}$ afeiçoada aos interesses da elite brasileira ${ }^{20}$, aquela que tem mantido há séculos a exploração de indivíduos não brancos, perpetuado e ampliado as desigualdades sociais' ${ }^{21}$.

As desvantagens observadas em relação às mulheres, principalmente as negras e indígenas ${ }^{18}$, estão associadas às relações de poder e dominação que penetram a vida social e cotidiana e organizam o funcionamento das instituições acadêmico-científicas. Sobre isso, cabe destacar que, no Brasil, para que as cientistas alcancem posições de destaque em CT\&I, como a bolsa PQ do CNPq, elas precisam, assim como os demais pesquisadores, submeter-se a um processo baseado em critérios gerais estabelecidos pelo CNPq e em critérios específicos, definidos pelo Comitê de Assessoramento (CA) de cada área de conhecimento ${ }^{22}$. Nesse processo, o CA é um elemento central já que é a partir do juízo emitido pelas(os) especialistas de cada área que é definida a concessão das bolsas para essas profissionais. 
No entanto, sua composição hegemonicamente masculina (somente $31 \%$ dos membros são mulheres) revela como as desigualdades de gênero presentes nos estágios mais avançados da carreira acadêmica precisam ser questionadas ${ }^{23}$. Os estudos de González Ramos ${ }^{24}$ e de Davyt e Velho ${ }^{25(9)}$ alertam que as desigualdades mantidas no interior dos sistemas científicos estão relacionadas com os discursos de 'objetividade' e de 'neutralidade' que moldam os processos de avaliação e reconhecimento de pesquisadoras e pesquisadores. Isso porque, ao defender cegamente esses princípios, continuadamente, tem-se escondido o lócus de enunciação dos sujeitos que decidem os rumos dos recursos científicos ${ }^{24}$, e essa condição tem provocado consequências bastante desvantajosas para as mulheres na carreira científica, principalmente no sentido de excluí-las dos processos de investigação e de negar-lhes(nos) autoridade epistêmica ${ }^{26}$.

Tomando tais críticas como referência, este artigo objetiva apresentar o panorama geral de distribuição das mulheres bolsistas PQ do CNPq nas várias áreas do conhecimento, detendo-se na área da psicologia, com vistas a problematizar as desigualdades existentes na ciência brasileira, especialmente nas ciências humanas e sociais.

\section{Material e métodos}

Este trabalho faz parte de um estudo mais amplo em nível de doutoramento no Programa de Pós-Graduação em Psicologia da Universidade Federal do Rio Grande do Norte (PPGPsi/UFRN) que investiga os impactos das desigualdades de gênero na ciência no cotidiano de mulheres pesquisadoras em psicologia. Este estudo foi aprovado pelo Comitê de Ética em Pesquisa da UFRN, sob o Certificado de Apresentação para Apreciação Ética - CAAE $\mathrm{n}^{\circ}$ 45268915.6.0000.5035.

A amostra do estudo incluiu 12.917 pesquisadoras(es) $\mathrm{PQ} / \mathrm{CNPq}$ das Ciências da Vida (CV), Engenharias, Ciências Exatas e da Terra
(Ecet) e Ciências Humanas e Sociais Aplicadas (CHSA), que corresponde ao universo total de bolsistas cadastrados no sistema PQ do CNPq, em julho de 2019. A amostra foi distribuída em dois conjuntos de dados: a) distribuição das(os) bolsistas nas CV [n=5.401]; nas Ecet [ $\mathrm{n}=4.181]$ e nas CHSA [n=3.335]); eb) distribuição das(os) bolsistas na área da psicologia [n=314]. Uma amostra adicional, não probabilística, de 85 mulheres bolsistas da área da psicologia, entre as 204 bolsistas PQ/CNPq cadastradas no sistema, também foi incluída neste estudo. A coleta da amostra adicional foi realizada a partir de formulário eletrônico cadastrado na plataforma SurveyMonkey, entre o período de abril e junho de 2020.

Para investigação da distribuição das cotas $\mathrm{PQ}$ referente às $\mathrm{CV}$, Ecet, CHSA, as variáveis categóricas nominais sexo, área de conhecimento, modalidade de bolsa e região foram comparadas por meio do teste não paramétrico qui-quadrado $\left(\mathrm{X}^{2}\right)$ no Statistical Package for the Social Sciences (SPSS v.22). Para análise dos dados respectivos à área de conhecimento psicologia, além das variáveis acima indicadas, adicionaram-se dados acerca da identificação étnico-racial das bolsistas, a partir das informações concedidas pelas próprias respondentes ao formulário on-line.

\section{Resultados e discussão}

\section{Perfil geral de distribuição de bolsa $\mathrm{PQ} / \mathrm{CNPq}$}

Identificou-se uma disparidade entre a quantidade de bolsas distribuídas por grande área do conhecimento e região do País, a julgar por: $[\mathrm{X} 2(2)=115.664 ; \mathrm{p}<0,00]$. Entre as 12.917 bolsas $\mathrm{PQ} / \mathrm{CNPq}$ existentes, a maior parte se concentra nas CV ( $\mathrm{n}=5.401)$, o que corresponde a $42 \%$, seguida das Ecet $(n=4.181)$, representando $32 \%$, e, por fim, das CHSA ( $n=3.335)$, área de menor prestígio na ciência nacional, que tem sofrido muitos ataques no atual governo, e que detém apenas $26 \%$ das cotas (tabela 1 ). 
Tabela 1. Distribuição das bolsas PQ/CNPq por grande área do conhecimento e região do País ( $n$, frequências, V-p, valor-p)

\begin{tabular}{lrrrrrrr}
\hline Grande área (CNPq) & Nordeste & Norte & Centro-Oeste & Sudeste & Sul & Total (n) & V-p \\
\hline Ciências da Vida & 560 & 116 & 378 & 3278 & 1069 & 5401 & 0 \\
$\begin{array}{l}\text { Engenharias, Ciências Exatas e da } \\
\text { Terra }\end{array}$ & 598 & 55 & 159 & 2625 & 748 & 4181 & 0 \\
$\begin{array}{l}\text { Ciências Humanas e Sociais Apli- } \\
\text { cadas }\end{array}$ & 375 & 82 & 189 & 1941 & 744 & 3335 & 0 \\
Total região (n) & 1533 & 253 & 726 & 7844 & 2561 & \\
\hline
\end{tabular}

Fonte: Elaboração própria.

A desvalorização das CHSA no processo de distribuição das cotas de bolsas PQ no Brasil é, na realidade, um padrão da trajetória científica nacional. Dados recuperados do CNPq informam que, entre 2001 e 2015, o órgão investiu um total de 163.198 bolsas PQ: 67.862 foram concedidas para CV, 59.628 para Ecet e somente 35.708 destinaram-se às CHSA, o que indica um histórico de investimento concentrado na primeira e na segunda, em prejuízo da última.

No cenário atual - diante da queda brutal do orçamento do $\mathrm{CNPq}^{27}$ que passou de $\mathrm{R} \$ 2,3$ bilhões em 2013 para R \$ 1,2 bilhão em 2019 e que "está menor do que esteve no início dos anos 2000, quando os fundos setoriais ainda estavam sendo criados"28(11), assim como da escalada da perseguição às CHSA pelo governo federal, com vistas a estrangular o já curto investimento na área -, acredita-se que o desprivilegio da CHSA tende a se tornar mais drástico. No que tange ao CNPq, sendo o principal órgão de fomento à pesquisa científica e tecnológica e incentivo à formação de pesquisadoras(es) brasileiras(os) do País, seu colapso implica que pesquisadoras(es), especialmente das CHSA, podem ficar sem fonte de recursos para financiar os projetos de pesquisa. Além do âmbito público, o setor produtivo é um importante investidor em CT\&I no Brasil. Porém, esse dificilmente vai financiar pesquisas que não gerem produtos de valor agregado aos interesses empresariais ${ }^{29}$.

A desvantagem das CHSA em relação às $\mathrm{CV}$ e Ecet na ciência nacional tem raízes alicerçadas na hierarquia entre os saberes que se estabeleceram no berço da ciência ocidental. Como se sabe, na ciência moderna, as ciências naturais, base disciplinar da CV e da Ecet, assumem, historicamente, uma posição de autoridade cognitiva ante as demais áreas de conhecimento. Nos dias atuais, dada a configuração da ciência em termos neoliberais, a lacuna, principalmente, entre as CV, área de maior investimento, e às CHSA, de menor prestígio, aumenta ainda mais tendo em vista que as ciências biológicas e biomédicas têm sido amplamente requisitadas em razão das possibilidades de produção de conhecimento voltada para produtos comerciais ${ }^{30}$.

As(os) pesquisadoras(es) das CHSA atuantes fora do eixo Sul-Sudeste se encontram em condições mais adversas, tendo em vista que a distribuição das bolsas PQ acompanha as diferenças existentes entre as regiões do País. Verificou-se que o eixo Sul-Sudeste, apesar das diferenças entre ambas as regiões, recebe, historicamente, mais recursos nas áreas da CV e Ecet. A região Nordeste ocupa o terceiro lugar, com uma distribuição mais equitativa entre as áreas, ainda que a maioria esteja nas CV e Ecet. Contudo, obtém número de bolsas indiscutivelmente inferior ao segundo lugar. As regiões Norte e Centro-Oeste recebem os menores recursos, com predomínio das CV e Ecet.

Esses dados mostram que as CHSA recebem o menor quantitativo de bolsas em todas as regiões do País e que a situação é ainda pior para as(os) bolsistas da CHSA que atuam no Nordeste, no Norte e no Centro-Oeste, regiões 
que sofrem as sequelas das estratégias desenvolvimentistas adotadas por governos brasileiros desde a década de 1930, que visavam à industrialização do Brasil e que resultaram em uma desigualdade das atividades produtivas - na região Sul e Sudeste - em relação às demais regiões ${ }^{31}$.

Desde 1960, teóricas feministas buscam descortinar os valores culturais da ciência ${ }^{3}$. Nessa busca, constatam que além da desigualdade entre as áreas de conhecimento, a ciência origina-se a partir de uma hierarquia de gênero que subalterniza a existência e dificulta a presença de mulheres na carreira científica. Sobre esse aspecto, os dados mostraram diferenças significativas entre mulheres e homens na distribuição das bolsas pelo País. A primeira conclusão do estudo é que as mulheres ainda são minoria na ciência brasileira. Do total de 12.917 bolsas, $8.316(64,4 \%)$ são ocupadas por homens; e somente 4.601 (35,6\%) das bolsas, pelas mulheres (tabela 2).

Tabela 2. Distribuição de mulheres e homens bolsistas $P Q / C N P q$ por grande área do conhecimento ( $n$, frequências, RA, Resíduos Ajustados, V-p, valor-p)

\begin{tabular}{|c|c|c|c|c|}
\hline Grandes áreas do conhecimento (CNPq) & Feminino $(n)$ & Masculino (n) & RA & V-p \\
\hline Ciências da Vida & 2.188 & 3.213 & $9.8 /-9.8$ & 0 \\
\hline Engenharia, Ciências Exatas e da Terra & 763 & 3.418 & $-28.5 / 28.5$ & 0 \\
\hline Ciências Humanas e Sociais Aplicadas & 1.650 & 1.685 & $19.4 /-19.4$ & 0 \\
\hline Total & 4.601 & 8.316 & 12.917 & \\
\hline \multicolumn{5}{|l|}{ Ciências da Vida (CV) } \\
\hline Área do conhecimento & $M(n)$ & $F(n)$ & Total (n) & $V-p$ \\
\hline Agronomia & 627 & 165 & 792 & 0 \\
\hline Biologia Geral & 1 & 5 & 6 & 0,02 \\
\hline Botânica & 119 & 95 & 214 & 0,01 \\
\hline Ciência e Tecnologia de Alimentos & 85 & 96 & 181 & 0 \\
\hline Educação Física & 76 & 18 & 94 & 0 \\
\hline Enfermagem & 11 & 169 & 180 & 0 \\
\hline Farmácia & 78 & 86 & 164 & 0 \\
\hline Farmacologia & 98 & 99 & 197 & 0 \\
\hline Fisiologia & 109 & 86 & 195 & 0,01 \\
\hline Fisioterapia e Terapia Ocupacional & 34 & 34 & 68 & 0,01 \\
\hline Fonoaudiologia & 3 & 51 & 54 & 0 \\
\hline Genética & 125 & 137 & 262 & 0 \\
\hline Imunologia & 76 & 99 & 175 & 0 \\
\hline Microbiologia & 85 & 107 & 192 & 0 \\
\hline Nutrição & 27 & 61 & 88 & 0 \\
\hline Parasitologia & 77 & 75 & 152 & 0 \\
\hline Recursos Florestais e Engenharia Florestal & 119 & 27 & 146 & 0 \\
\hline Recursos Pesqueiros e Engenharias de Pesca & 80 & 31 & 111 & 0,09 \\
\hline Saúde Coletiva & 99 & 114 & 213 & 0 \\
\hline Zoologia & 171 & 55 & 226 & 0 \\
\hline Zootecnia & 197 & 65 & 262 & 0 \\
\hline
\end{tabular}


Tabela 2. (cont.)

\begin{tabular}{|c|c|c|c|c|}
\hline Grandes áreas do conhecimento $(\mathrm{CNPq})$ & Feminino $(n)$ & Masculino ( $n$ ) & RA & V-p \\
\hline \multicolumn{5}{|c|}{ Engenharia, Ciências Exatas e da Terra (Ecet) } \\
\hline Área do conhecimento & $M(n)$ & $F(n)$ & Total (n) & $V-p$ \\
\hline Astronomia & 85 & 27 & 112 & 0,01 \\
\hline Engenharia Aeroespacial & 51 & 11 & 62 & 0 \\
\hline Engenharia Biomédica & 57 & 4 & 61 & 0 \\
\hline Engenharia Civil & 227 & 59 & 286 & 0 \\
\hline Engenharia de Minas & 21 & 3 & 24 & 0,02 \\
\hline Engenharia de Produção & 116 & 37 & 153 & 0 \\
\hline Engenharia Elétrica & 272 & 18 & 290 & 0 \\
\hline Engenharia Mecânica & 264 & 32 & 296 & 0 \\
\hline Engenharia Naval e Oceânica & 10 & 0 & 10 & 0,02 \\
\hline Física & 890 & 80 & 970 & 0 \\
\hline Matemática & 313 & 26 & 339 & 0 \\
\hline Oceanografia & 96 & 31 & 127 & 0,01 \\
\hline Probabilidade e Estatística & 55 & 14 & 69 & 0,01 \\
\hline Química & 507 & 203 & 710 & 0 \\
\hline \multicolumn{5}{|c|}{ Ciências Humanas e Sociais Aplicadas (CHSA) } \\
\hline Área do conhecimento & $M(n)$ & $F(n)$ & Total (n) & $V-p$ \\
\hline Administração & 140 & 56 & 196 & 0,04 \\
\hline Antropologia & 73 & 85 & 158 & 0 \\
\hline Arquitetura e Urbanismo & 52 & 57 & 109 & 0 \\
\hline Artes & 47 & 67 & 114 & 0 \\
\hline Ciência da Informação & 22 & 28 & 50 & 0 \\
\hline Comunicação & 67 & 65 & 132 & 0 \\
\hline Economia & 180 & 29 & 209 & 0 \\
\hline Educação & 144 & 253 & 397 & 0 \\
\hline Filosofia & 120 & 32 & 152 & 0 \\
\hline História & 136 & 119 & 255 & 0 \\
\hline Letras & 107 & 131 & 238 & 0 \\
\hline Linguística & 67 & 165 & 232 & 0 \\
\hline Planejamento Urbano e Regional & 43 & 43 & 86 & 0,01 \\
\hline Psicologia & 110 & 204 & 314 & 0 \\
\hline Serviço Social & 11 & 69 & 80 & 0 \\
\hline Turismo & 4 & 13 & 17 & 0 \\
\hline
\end{tabular}

Fonte: Elaboração própria.

Desde que Rossi ${ }^{32}$ questionou 'por que tão poucas?', a ausência e a invisibilidade das mulheres na ciência vêm-se consolidando como uma temática recorrente nos estudos de Gênero, pois, apesar das mudanças nesses padrões apontadas pela Elsevier ${ }^{9}$, a sub-representação de pesquisadoras na carreira científica é um fenômeno atual, no âmbito 
internacional e nacional. Dados recentes revelam que as mulheres representam uma parcela minoritária na ciência mundial, com uma média regional de participação de $41,5 \%$ nos países Árabes, 39,3\% na Europa Central e na Oriental, $48,2 \%$ na Ásia Central, $23,9 \%$ na Ásia Oriental e no Pacífico, 45,1\% na América Latina e no Caribe, $32,7 \%$ na América do Norte e na Europa Ocidental, 18,5\% no Sul e no Oeste da Ásia, e $31,8 \%$ na África Subsaariana ${ }^{5}$. Em anos anteriores, os estudos de Fox ${ }^{33}$ nos Estados Unidos; de Musselin ${ }^{34}$ na França e na Alemanha; de Probert ${ }^{35}$ na Austrália; de Gupta e Sharma ${ }^{36}$ na Índia, e de Ma et al. ${ }^{37}$ na China constataram que as mulheres eram minoria entre pesquisadores combolsas de pesquisa e que a desvantagem delas persistia mesmo quando possuíam nível educacional elevado. No Brasil, a presença feminina é preponderante entre os matriculados na formação graduada e pós-graduada, representando $57,2 \%{ }^{15}$ e $54 \%{ }^{18}$ respectivamente. No entanto, no âmbito do CNPq, ocuparam, aproximadamente, $36 \%$ das bolsas entre 2013 e 2017 (35,64\% em $2013 ; 35 \%, 36 \%$ em 2014; 35,19\% em 2015; 35,37\% em 2016; e 35,32\% em 2017) ${ }^{38}$.

A segunda conclusão oriunda da análise dos dados é o fato de as(os) pesquisadoras(es) se concentrarem em determinadas áreas do conhecimento, considerando: [X2 (2) = 885.663; $\mathrm{p}<0,00]$ (tabela 2). Na tabela 2, demonstram-se as áreas de conhecimento da CV, Ecet e CHSA em que se detectou uma diferença significativa entre mulheres e homens. A avaliação da distribuição das(os) bolsistas na CV revelou uma assimetria de gênero significativa. Por intermédio do teste Bonferroni, detectaram-se diferenças em 21 cursos: [ $\mathrm{X}^{2}(2)=2206,15^{\mathrm{a}}$; $\mathrm{p}<0,00]$. O número de mulheres foi inferior ao esperado na distribuição de Cursos hegemonicamente frequentados por homens, a exemplo da Agronomia ( $p<0,00)$, Recursos Florestais e Engenharia Florestal $(p<0,00)$ e Zootecnia $(p<0,00)$, entre outros. Já o número de homens foi inferior em 14 Cursos, entre os quais, Enfermagem $(p<0,00)$, Fisioterapia e Terapia Ocupacional $(p<0,01)$, Fonoaudiologia $(p<0,00)$ e Saúde Coletiva $(p<0,00)$.
A análise apontou o mesmo resultado em algumas áreas das Ecet, tendo em vista: $\left[\mathrm{X}^{2}\right.$ $(2)=2206,75 ; p<0,00]$. Nesse caso, a contagem esperada e observada foi desfavorável para as mulheres. Posteriormente, o teste post hoc Bonferroni revelou que essas diferenças significativas se encontravam, especificamente, nos Cursos de Astronomia ( $p<0,01)$, Física $(p<0,00)$ e nas Engenharias de uma geral, entre outros.

Nas CHSA, uma diferença estatisticamente significativa entre sexo e áreas de conhecimento também foi encontrada: $\left[\mathrm{X}^{2}(2)=\right.$ $\left.2206,75^{a} ; \mathrm{p}<0,00\right]$. A análise revelou que 16 áreas contribuíram para essa distribuição sem equidade, indicando que há uma concentração de mulheres nos Cursos de Antropologia, Arquitetura e Urbanismo, Artes, Ciência da Informação, Comunicação, Educação, História, Letras, Linguística, Psicologia, Serviço Social e Turismo. Por outro lado, o número de mulheres foi menor ao esperado em Administração $(p<0,04)$, Economia $(p<0,00)$ e Filosofia $(p<0,00)$, Cursos com quantitativo elevado de bolsistas homens.

Os resultados das CV, Ecet e CHSA indicam uma proporção mais alta de mulheres bolsistas em áreas profissionais historicamente associadas ao trabalho doméstico, familiar, reprodutivo e ao cuidado. Por outro lado, observa-se a concentração de homens em disciplinas científicas ligadas à política, ao setor econômico e às ciências da natureza, o que remonta, de acordo com Olinto ${ }^{\mathbf{3 9}}$; Tabak ${ }^{\mathbf{1 1}}$; Leta $^{\mathbf{1 2}}$, para uma segregação horizontal, ou seja, uma proporção maior de um dos sexos em algumas áreas profissionais.

Dados publicados pela Elsevier ${ }^{9}$ diagnosticaram um padrão semelhante em diferentes países e regiões. As mulheres são mais bem representadas nas áreas temáticas da bioquímica, genética, biologia molecular, imunologia, microbiologia, medicina, enfermagem e psicologia, sendo, pelo menos, $40 \%$ do total pesquisadores de cada área. De modo oposto, em ciência da computação, energia, engenharia, matemática, física e astronomia, os homens são predominantes, tendo em vista que são 
áreas associadas à racionalidade, à razão, valor socialmente construído como masculino em contraponto às áreas relacionadas socialmente com a 'natureza feminina'.

A terceira conclusão do estudo é que há uma desigualdade na distribuição entre homens e mulheres, levando em conta a modalidade de bolsa PQ (tabela 3). Ao analisar a distribuição geral dos(as) bolsistas PQ/CNPq por sexo e modalidade de bolsa, constatou-se mais uma diferença significativa: as mulheres detêm somente $35,6 \%$ do total de cotas (tabela 3). Verifica-se também que as pesquisadoras são minoria em todas as modalidades, considerando: $\left[\mathrm{X}^{2}(2)=52944,0 ; \mathrm{p}<0,00\right]$. Além disso, elas estão em desvantagem significativa em comparação aos bolsistas homens no nível PQ-2, $(\mathrm{p}<0,00)$ e nos níveis mais privilegiados da carreira: PQ-SR, $(\mathrm{p}<0,02), P Q-1 A,(p<0,00)$ e PQ-1B, $(p<0,02)$.

Tabela 3. Distribuição de mulheres e homens bolsistas $\mathrm{PQ} / \mathrm{CNPq}$ das grandes áreas do conhecimento por modalidade de bolsa ( $n$, frequências, $\vee-p$, valor-p)

\begin{tabular}{|c|c|c|c|c|c|c|c|c|}
\hline Modalidade de Bolsa & & PQ-SR & $P Q-1 A$ & PQ-1B & PQ-1C & PQ-1D & $P Q-2$ & Total \\
\hline Feminino & $n$ & 43 & 310 & 354 & 453 & 754 & 43 & 4.601 \\
\hline \multirow[t]{2}{*}{ Masculino } & $n$ & 117 & 822 & 741 & 753 & 1.316 & 117 & 8.316 \\
\hline & $V-p$ & 0,02 & 0,00 & 0,02 & 0,13 & 0,42 & 0,00 & \\
\hline Total & & & & & & & & 12.917 \\
\hline \multicolumn{9}{|l|}{ Ciências da Vida (CV) } \\
\hline Feminino & $\mathrm{n}$ & 19 & 131 & 166 & 224 & 379 & 1.264 & 2.188 \\
\hline \multirow[t]{2}{*}{ Masculino } & $n$ & 35 & 368 & 289 & 301 & 564 & 1.656 & 3.213 \\
\hline & $V-p$ &, 42 &, 00 &, 07 &, 27 &, 84 &, 00 & \\
\hline Total & & & & & & & & 5.401 \\
\hline \multicolumn{9}{|c|}{ Engenharia, Ciências Exatas e da Terra (Ecet) } \\
\hline Feminino & $n$ & 5 & 32 & 48 & 78 & 121 & 479 & 763 \\
\hline \multirow[t]{2}{*}{ Masculino } & $n$ & 61 & 318 & 315 & 311 & 529 & 1.884 & 3.418 \\
\hline & $V-p$ &, 02 &, 00 &, 01 &, 32 &, 76 &, 00 & \\
\hline Total & & & & & & & & 4.181 \\
\hline \multicolumn{9}{|c|}{ Ciências Humanas e Sociais Aplicadas (CHSA) } \\
\hline Feminino & $n$ & 19 & 147 & 140 & 151 & 254 & 939 & 1.650 \\
\hline \multirow[t]{2}{*}{ Masculino } & $\mathrm{n}$ & 21 & 136 & 137 & 141 & 223 & 1.027 & 1.685 \\
\hline & $V-p$ &, 76 &, 37 & ,69 &, 42 &, 07 & ,02 & \\
\hline Total & & & & & & & & 3.335 \\
\hline
\end{tabular}

Fonte: Elaboração própria.

Na análise da distribuição das(os) bolsistas por modalidade de bolsa em cada grande área de conhecimento, identificou-se que as mulheres têm experimentado desvantagens, sobretudo, nas Ecet e nas CV, áreas com maioria dos recursos no País. Nas Ecet, a desvantagem das mulheres ocorre - especificamente - nos níveis PQ-2 $(\mathrm{p}<0,00), P Q-1 B(p<0,01), P Q-1 A$ $(p<0,00)$ e $P Q-S R(p<0,02)$; e nas CV, acontece nos níveis PQ-2 $(\mathrm{p}<0,00)$ e PQ-1A $(\mathrm{p}<0,00)$. Desse modo, elas enfrentam dificuldades tanto na entrada do sistema quanto para alcançar as modalidades de maior prestígio. Já nas CHSA, área com menor movimentação de recursos, 
uma diferença estatística em desfavor das mulheres foi constatada apenas na modalidade PQ-2 ( $<<0,00)$, ou seja, no acesso ao sistema PQ do CNPq.

Segundo Valentova, Otta, Silva et al. ${ }^{4}$ e Leta $^{12}$, no Brasil, as mulheres enfrentam condições desiguais que as impedem de alcançar postos mais prestigiados do âmbito científico, como os níveis sêniores das bolsas PQ, modalidade de acesso a maiores subsídios, bem como de integrar equipes de comitês deliberativos e diretoria executiva, responsáveis pela decisão em termos da concessão de recursos para pesquisa e formação de pesquisadores. Em época de redução drástica do orçamento destinado à ciência, as mulheres bolsistas serão ainda mais prejudicadas. Além de driblar o curto orçamento do CNPq, principalmente, em áreas com menor investimento e/ou menor participação feminina, elas enfrentarão disputas, cada vez mais acirradas, para se inserir, permanecer e progredir no sistema PQ.

Outras investigações acerca da participação das mulheres na ciência nacional, desde a década de 1990, têm identificado como a sub-representação das mulheres, principalmente, nos níveis mais privilegiados da carreira, está ancorada aos saberes androcêntricos que estereotiparam/estereotipam e violentaram/ violentam as mulheres, forjados pelo homem ocidental, branco, heterossexual e pertencente às classes privilegiadas ${ }^{40}$.

\section{Perfil de distribuição de bolsa $\mathrm{PQ}$ na psicologia}

A psicologia - como parte das CHSA no âmbito do CNPq - sofre os efeitos das desigualdades presentes na distribuição das cotas entre as grandes áreas. As CHSA, como indicado anteriormente, detêm apenas $26 \%$ do total de bolsas PQ. Essas cotas estão distribuídas da seguinte forma nas regiões do País: Sudeste $(\mathrm{n}=1.941 ; 58,2 \%) ; \operatorname{Sul}(\mathrm{n}=748 ; 22,4 \%)$; Nordeste $(\mathrm{n}=375 ; 11,2 \%)$; Centro-Oeste $(\mathrm{n}=189 ; 5,7 \%)$, Norte ( $\mathrm{n}=82 ; 2,5 \%)$, ou seja, seguem o padrão da distribuição geral pelo Brasil.
No que diz respeito à análise da distribuição das cotas nas CHSA por sexo, o teste não indicou uma diferença estatística significativa, porém, notou-se que do total de 3.335 bolsas, os homens ocupam 1.685 (50,5\%), e as mulheres detêm 1.650 (49,5\%), cenário que indica uma vantagem em favor dos homens. Embora as CHSA sejam uma área marcada pela existência de nichos disciplinares 'femininos' e pela importante inserção de mulheres, a análise da distribuição das(os) bolsistas por modalidade de bolsa também anunciou alguma vantagem para os homens. Identificou-se que as mulheres são maioria em quase todos os níveis (PQ-2, PQ-1D, PQ-1C, $P Q-1 B$ e $P Q-1 A)$, mas enfrentam dificuldades para acessar a modalidade PQ-SR, modalidade de bolsa independente, direcionada as(os) cientistas que são lideranças em seus campos de atuação e destaque entre seus pares, por pelo menos 20 anos $^{31}$.

Internamente à grande área, a psicologia detém o segundo maior número de cotas $(n=314)$, ficando atrás somente da educação $(n=397)$, como pode ser visto na tabela 2. Isso corresponde a quase $10 \%$ do total de bolsas das CHSA. A distribuição das bolsas sofre os efeitos das desigualdades de recursos por região do País demonstradas no padrão da disposição das bolsas em âmbito nacional e, especificamente, nas CHSA, assim apresentada: Sudeste ( $\mathrm{n}=155 ; 49,4 \%) ; \operatorname{Sul}(\mathrm{n}=75 ; 23,9 \%)$; Nordeste ( $\mathrm{n}=50 ; 15,9 \%)$; Centro-Oeste $(\mathrm{n}=20$; $6,4 \%)$; Norte ( $\mathrm{n}=14 ; 4,5 \%)$.

Ao analisar mais detidamente, levando em conta a distribuição por sexo e modalidade da bolsa, percebe-se que as mulheres representam quase o dobro dos homens em termos quantitativos, porém, trata-se de uma área tradicionalmente ocupada pelas mulheres. Há também uma distribuição de pesquisadoras em todas as modalidades de bolsa PQ, tal como já documentado por Weber, Ramos, Mester et al. ${ }^{\mathbf{1 1}}$. No entanto, nota-se que a vantagem das mulheres parece ser menor no topo da carreira, notadamente nas modalidades PQ1A e PQ1B (tabela 4). 
Tabela 4. Distribuição de mulheres e homens bolsistas PQ/CNPa por modalidade de bolsa na psicologia (n, frequências, RA, Resíduos Ajustados)

\begin{tabular}{lrrrrrrrr}
\hline Modalidade de Bolsa & & PQ-SR & PQ-1A & PQ-1B & PQ-1C & PQ-1D & PQ-2 & Total \\
\hline Feminino & $\mathrm{n}$ & 2 & 18 & 9 & 19 & 38 & 118 & 204 \\
& $\mathrm{RA}$ & 1.0 & -1.3 & -.4 & 1.2 & 1.8 & -1.2 & \\
Masculino & $\mathrm{n}$ & 0 & 15 & 6 & 6 & 12 & 71 & 110 \\
& $\mathrm{RA}$ & -1.0 & 1.3 & .4 & -1.2 & -1.8 & 1.2 & \\
Total & & 2 & 33 & 15 & 25 & 50 & 189 & 314 \\
\hline
\end{tabular}

Fonte: Elaboração própria.

Esse cenário está associado a um conjunto de determinantes históricos e sociais e se aplica às mulheres pesquisadoras de todas as áreas. Contudo, não se pode esquecer que a psicologia se constituiu como campo teórico-prático, associado às elites brasileiras. Nesse sentido, ocupado predominantemente por mulheres brancas das classes mais favorecidas. Assim, em meio a um conjunto de desvantagens que atingem, notadamente, mulheres negras e indígenas ${ }^{18}$, o acesso à educação superior e pós-graduada não se constituiu um obstáculo para a maior parte daquelas que ingressaram na psicologia.

Independentemente dessa condição, a conciliação entre o tempo dedicado à ciência e à família tem sido apontado como um dos principais aspectos que dificultam a inserção, a permanência e a progressão das mulheres na carreira ${ }^{42}$. Segundo Santos ${ }^{43}$, o tempo de permanência no exercício do trabalho científico não é suficiente para o cumprimento de todas as atividades exigidas para esses níveis de excelência acadêmica. Em função disso, a extensão do trabalho científico para o âmbito doméstico emerge como uma necessidade e realidade no cotidiano das mulheres.

Em seu estudo, Santos ${ }^{43}$ observou que o tempo dedicado à ciência representa, para algumas pesquisadoras, a negação do cuidado para com o(s/as) companheiro(s/as) e filho(s/as). A pesquisa realizada pelo Parent in Science ${ }^{23}$ demonstrou que pesquisadoras com filhos em idade escolar não conseguem manter o ritmo de produção científica devido às tarefas do âmbito doméstico e familiar ${ }^{44}$. Embora a responsabilidade do trabalho doméstico esteja historicamente associada à mulher, devido à biologização da 'vocação para o lar' socialmente construída, Collins ${ }^{45}$ alerta que mulheres brancas e negras experenciam de formas distintas esse estereótipo.

É necessário, entretanto, remontar a um período anterior à formação do mercado de trabalho assalariado brasileiro para compreender a participação das mulheres negras no mundo do trabalho e no lar, já que desde o período escravista, muito antes do ingresso do grande contingente de mulheres brancas de classes populares, na segunda metade do século $X X$, as primeiras já estavam submetidas à exploração do trabalho46(103).

Em razão disso, buscou-se conhecer como se configura essa realidade dentre as mulheres pesquisadoras na psicologia. $\mathrm{Na}$ análise do perfil étnico-racial das mulheres bolsistas da área da psicologia, obtido por meio de questionário e da autorreferenciação, verificou-se que há uma desigualdade de raça/etnia, conforme a tabela 5. Na distribuição de bolsas, identificou-se que $82,35 \%$ das bolsistas são brancas, $10,59 \%$ são pardas, $4,71 \%$ são pretas e $1,18 \%$ são amarelas. De 85 mulheres respondentes, não houve nenhuma referência a raça/etnias indígenas. Observa-se que apesar da expansão do ensino superior brasileiro e das políticas 
afirmativas, desde os anos 2000, ainda há uma significativa sub-representação de estudantes negras(os) na área desde a graduação, a qual se intensifica em estágios educacionais mais avançados, e que está associada às desigualdades educacionais impostas à população não branca e à alta concorrência para ingressar no Curso superior de Psicologia ${ }^{47}$.

Tabela 5. Distribuição das mulheres bolsistas PQ/CNPq da área psicologia por identificação étnico-racial ( $n$, frequência, $\%$ porcentagem)

\begin{tabular}{lrr}
\hline Raça/Etnia & (n) & (\%) \\
\hline Branca & 70 & $82,35 \%$ \\
Parda & 9 & $10,59 \%$ \\
Preta & 4 & $4,71 \%$ \\
Amarela & 1 & $1,18 \%$ \\
Outra & 1 & $1,18 \%$ \\
Total & 85 & $100 \%$ \\
\hline
\end{tabular}

Fonte: Elaboração própria.

A não presença das mulheres negras e indígenas constatada na área da psicologia tem suas raízes na violenta classificação social e epistêmica do projeto moderno colonial que se mantém atual em função da colonialidade de poder $^{16}$, saber ${ }^{48}$ e ser ${ }^{49}$. Segundo Quijano ${ }^{16(1)}$, a colonialidade é um dos eixos constitutivo e específico do padrão de poder atual que se alicerça na imposição de uma classificação da população a partir da ideia de raça, "uma construção mental que expressa a experiência básica da dominação colonial" e que desde então permeia cada dimensão da existência social: 1) trabalho e seus produtos; 2) natureza e seus recursos de produção; 3) sexo e seus produtos e a reprodução da espécie; 4) subjetividade e seus produtos; e 5) autoridade e seus instrumentos.

Esse padrão de poder colonial forjou identidades, hierarquias e estabeleceu o papel de cada raça dentro da sociedade ocidental ${ }^{43}$. A conformação desses papéis, porém, não se construiu - originariamente - somente pelo aspecto da classificação étnico-racial, mas também pela imposição estrutural de gênero, que, por sua vez, interliga-se intimamente à primeira. Lugones ${ }^{17}$ defende, fundamentada nas discussões das intelectuais
Oyéronké Oyewùmi e Paula Gunn Allen, que tanto raça quanto gênero foram introduzidos pelo sistema moderno/colonial. A partir de Oyewùmi, Lugones ${ }^{17}$ compreende que $o$ gênero foi introduzido pelo elemento colonial, uma vez que não havia sistema de gênero nas sociedades pré-intrusão, como é o caso da sociedade africana yorubá. Nessa sociedade, não havia divisão macho/fêmea binária e hierarquizada. Foi a partir da intrusão colonial que as fêmeas foram rotuladas como mulheres e as africanas e africanos foram rotulados pela raça - uma construção intersubjetiva que teve como consequência a dupla subordinação das mulheres africanas ${ }^{\mathbf{1 7 ( 8 8 )}}$, mecanismo que se estendeu para todas as mulheres não-brancas.

As sequelas dessa injustiça social e epistêmica no campo da ciência são visíveis em maior intensidade entre as mulheres-pesquisadoras negras e indígenas, uma vez que são vítimas do que Sueli Carneiro ${ }^{50}$ denomina por epistemicídio. Segundo a autora, o epistemicídio, para além da invisibilidade e desqualificação do conhecimento dos povos subjugados no período colonial, trata-se de um processo persistente de produção de pobreza cultural, seja pela negação ao acesso à educação de qualidade, pela produção da inferiorização 
*Orcid (Open Researcher and Contributor ID). intelectual; pelos diferentes mecanismos de deslegitimação do outro como portador e produtor de conhecimento e de rebaixamento da capacidade cognitiva pela carência material e/ou pelo comprometimento da autoestima pelos processos de discriminação correntes no processo educativo.

Isso porque, não é possível desqualificar as formas de conhecimento dos povos dominados sem desqualificá-los também, individual e coletivamente, como sujeitos cognoscentes. E, ao fazê-lo, destitui-Ihe a razão, a condição para alcançar o conhecimento 'legítimo' ou legitimado. Por isso, o epistemicídio fere de morte a racionalidade do subjugado ou a sequestra e mutila a capacidade de aprender etc. É uma forma de sequestro da razão, em duplo sentido: pela negação da racionalidade do Outro ou pela assimilação cultural que em outros casos lhe é imposta. Sendo, pois, um processo persistente de produção da inferioridade intelectual ou da negação da possibilidade de realizar as capacidades intelectuais ${ }^{50(107)}$

Dada tal configuração, as mulheres negras, assim como as mulheres indígenas, dificilmente ocupam posições de destaque na ciência, tal como constado na distribuição das bolsas da psicologia. Isso traz repercussões importantes à produção de conhecimento na área e à manutenção dessas mulheres à margem do sistema científico. A possibilidade de uma maior diversidade étnico-racial na ciência brasileira, em particular na psicologia, esbarra, principalmente, na resistência do sistema científico que reproduz a lógica dos países centrais, os padrões androcêntricos, o racismo estrutural, as hierarquias sociais de gênero. Dessa maneira, ao persistir uma lógica de avaliação discriminatória, que se autorreconhece como objetiva e neutra, acaba-se por ocultar as variadas opressões e desigualdades existentes na trajetória acadêmico-científica de mulheres e homens e as disparidades latentes entre mulheres brancas, negras e indígenas.

\section{Considerações finais}

A distribuição de bolsas PQ por área de conhecimento do CNPq revela as diferenças abissais entre as regiões do País. Há uma concentração de bolsas nas regiões Sul e Sudeste. É nelas que há um maior número de bolsistas das disciplinas das CV e Ecet, áreas que, em função da linguagem e dos métodos autorreconhecidos como neutros e universais, gozam de maior privilégio na ciência moderna. Embora a seleção das(os) bolsistas PQ contemple condições e objetivos particulares de cada área de conhecimento de modo a promover uma triagem justa e igualitária, os dados apontam que as desigualdades de gênero na ciência persistem em pelos menos duas direções: a) mulheres e homens se concentram em diferentes disciplinas do conhecimento; eb) mulheres enfrentam dificuldades tanto para acessar o sistema PQ quanto para alcançar as modalidades de bolsa de maior prestígio científico.

Na psicologia, apesar da presença das mulheres em todas as modalidades de bolsa, elas ocupam menos posições no topo da carreira, notadamente, nas modalidades PQ1A e PQ1B. Ademais, há um padrão de desigualdade de raça/etnia entre as bolsistas PQ da psicologia. Identificou-se que a grande maioria das bolsistas são brancas, poucas negras e que não há pesquisadoras indígenas. A invisibilidade das mulheres negras e indígenas, constatada na área da psicologia - e, certamente, em todas as áreas do conhecimento -, tem suas raízes na classificação social e epistêmica imposta pelo projeto moderno colonial, que se mantém na atualidade do sistema científico brasileiro.

\section{Colaboradoras}

Cunha R (0000-0003-0201-5436)*, Dimenstein M (0000-0002-5000-2915)* e Dantas C (00000003-4778-9400)* contribuíram igualmente para a elaboração do manuscrito. 


\section{Referências}

1. Harding S. Ciencia y feminismo. Madrid: Morata; 1996.

2. Keller EF, Longino HE, editores. Feminism \& Science. New York: Oxford University Press; 1996.

3. Barros SCV, Mourão L. Desenvolvimento na carreira de bolsistas produtividade: uma análise de gênero. Arq. bras. psicol. 2019 [acesso em 2020 nov 8]; 71(2):68-83. Disponível em: http://pepsic.bvsalud.org/scielo.php?script=sci arttext\&pid=S1809-52672019000200006\&lng=pt.

4. Valentova JV, Otta E, Silva ML, et al. Underrepresentation of women in the senior levels of Brazilian science. PeerJ. 2017 [acesso em $2018 \mathrm{dez}$ 10]; (5):e4000. Disponível em: https://doi.org/10.7717/peerj.4000.

5. Organização das Nações Unidas para a Educação, a Ciência e a Cultura. Women in Science. Paris: Unesco; Institute for Statistics; 2019. [acesso em 2019 jul 2]. Disponível em: http://uis.unesco.org/sites/default/files/documents/fs55-women-in-science-2019-en.pdf.

6. Mason M, Ekman E. Mothers on the fast track: How a new generation can balance family and careers. New York: Oxford University Press; 2007. [acesso em 2019 fev 5]. Disponível em: https://oxford.universitypressscholarship.com/view/10.1093/acprof:o so/9780195182675.001.0001/acprof-9780195182675.

7. Alonso A, Diz I, Lois M. Is gender mainstreaming helping women scientists? Evidences from research policies in Spain. INFE. 2016 [acesso em 2019 fev 5]; 7(2):27391. Disponível em: https://revistas.ucm.es/index.php/ INFE/article/view/52963.

8. Bargillole B, Goode J. The Contradiction of the Myth of Individual Merit, and the Reality of a Patriarchal Support System in Academic Careers. Europ J. Women's Stud. 2001 [acesso em 2019 mar 12]; 8(2):161180. Disponível em: https://journals.sagepub.com/ doi/10.1177/135050680100800203.

9. Elsevier. Gender in the Global Research Landscape Re- port. Amsterdam: Elsevier; 2017. [acesso em 2018 mar 2]. Disponível em: https://www.elsevier.com/research-intelligence/resource-library/gender-report.

10. Elsevier. The Researcher Journey Through a Gender Len. Amsterdam: Elsevier; 2020. [acesso em 2021 fev 20]. Disponível em: https://www.elsevier.com/_data/assets/ pdf file/0011/1083971/Elsevier-gender-report-2020.pdf.

11. Tabak F. O Laboratório de Pandora: estudos sobre a ciência no feminino. Rio de Janeiro: Garamond; 2002.

12. Leta J. As mulheres na ciência brasileira: crescimento, contrastes e um perfil de sucesso. Estud. Avanç. 2003 [acesso em 2018 maio 5]; 17(49):271-284. Disponível em: https://doi.org/10.1590/S0103-40142003000300016.

13. Vasconcellos E, Brisolla S. Presença feminina no estudo e no trabalho da ciência na Unicamp. Cad. Pagu. 2009 [acesso em 2018 maio 5]; (32):215-265. Disponível em: https://doi.org/10.1590/S0104-83332009000100008.

14. Velho L, León E. A construção social da produção científica por mulheres. Cad. Pagu. 2012 [acesso em 2019 jul 21]; (10):309-44. Disponível em: https://periodicos.sbu.unicamp.br/ojs/index.php/cadpagu/article/view/4631474.

15. Brasil. Ministério da Educação. Notas Estatísticas - Censo da Educação Superior 2016. Brasília, DF: INEP; 2016. [acesso em 2020 fev 2]. Disponível em: http://download.inep.gov.br/educacao_superior/censo_superior/documentos/2016/notas_sobre_o_censo_da_educacao_superior_2016.pdf.

16. Quijano A. Colonialidade do poder, eurocentrismo e América Latina. In: Quijano A. A colonialidade do saber: eurocentrismo e ciências sociais. Perspectivas latino-americanas. Buenos Aires: CLACSO; 2005. [acesso em 2018 mar 2]. Disponível em: http://bibliotecavirtual. clacso.org.ar/clacso/sur-sur/20100624103322/12_Quijano.pdf.

17. Lugones M. Colonialidad y género. Tabula Rasa. 2008 [acesso em 2018 mar 16]; (9):73-101. Disponível em: ht- 
tps://www.revistatabularasa.org/numero-9/05lugones. pdf.

18. Coordenação de Aperfeiçoamento de Pessoal de Nível Superior. Discentes da Pós-Graduação stricto sensu do Brasil [2017 a 2020]. Brasília, DF: Ministério da Educação; CAPES; 2018. [acesso em 2020 ago 2]. Disponível em: https://dadosabertos.capes.gov.br/dataset/2017-2020-discentes-da-pos-graduacao-stricto-sensu-do-brasil.

19. Lhullier L, organizador. Quem é a psicóloga brasileira? Brasília, DF: Conselho Federal de Psicologia - CFP; 2013. [acesso em 2018 ago 2]. Disponível em: https://site.cfp. org.br/publicacao/quem-e-a-psicologa-brasileira/.

20. Bock AMB. Psicologia e sua ideologia: 40 anos de compromisso com as elites. In: Bock AMB, organizador. Psicologia e o compromisso social. São Paulo: Cortez; 2003.

21. Souza J. A elite do atraso: da escravidão a Bolsonaro. Rio de Janeiro: Estação Brasil; 2019.

22. Wendt GW, Lisboa CSM, De Sousa DA, et al. Perfil dos bolsistas de produtividade em pesquisa do CNPq em Psicologia. Psicol. Ciênc. Prof. 2013 [acesso em 2018 ago 2]; 33(3):536-547. Disponível em: https://doi.org/10.1590/ S1414-98932013000300003.

23. Parent in Science. Produtividade acadêmica durante a pandemia: efeitos de gênero, raça e parentalidade. 2020. [acesso em 2021 jan 2]. Disponível em: https://327b604e5cf4-492b-910b-e35e2bc67511.filesusr.com/ugd/ob341 b_81cd8390d0f94bfd8fcd17ee6f29bc0e.pdf?index=true.

24. González Ramos A, Benavente B. Excelencia en la ciencia: una reflexión crítica afirmativa. Cad. Pesqui. 2017 [acesso em jan 2020 10]; 47(166):1372-1394. Disponível em: https://doi.org/10.1590/198053144233.

25. Davyt A, Velho L. A avaliação da ciência e a revisão por pares: passado e presente. Como será o futuro? Hist. cienc. saúde-Manguinhos. 2000 [acesso em 2020 ago 26]; 7(1):93-116. Disponível em: https://doi.org/10.1590/ S0104-59702000000200005.

26. Sardenberg C. Da Crítica Feminista à Ciência a uma Ciência Feminista? In: Costa AAA, Sardenberg C. Feminis- mo, ciência e tecnologia. Salvador: Universidade Federal da Bahia; Faculdade Filosofia e Ciências Humanas; 2002.

27. Conselho Nacional de Desenvolvimento Científico e Tecnológico. Dados abertos - Planilha de investimento em bolsas de produtividade de pesquisa de 2001 a 2015 do CNPq. [acesso em 2019 jan 2]. Disponível em: http:// www.cnpq.br/web/guest/apresentacaol.

28. Gamboa Solís FM, Pérez Abreu AM. Tiempo de academiay el poder 'poder' de las mujeres en el desafío familia-trabajo. Las académicas de la Universidad Michoacana de San Nicolás de Hidalgo. La ventana. Rev. estud. Gén. 2017 [acesso em 2018 jun 2]; 5(45):241-268. Disponível em: http://www.scielo.org.mx/scielo.php?script=sci arttext\&pid=S1405-94362017000100241\&lng=es\&tlng $=$ es.

29. Oliveira A. Política científica no Brasil: análise das políticas de fomento à pesquisa do CNPq. [dissertação]. Florianópolis: Universidade Federal de Santa Catarina; 2003. 150 p. [acesso em 2019 nov 22]. Disponível em: https:// repositorio.ufsc.br/xmlui/handle/123456789/85078.

30. Lander E. La ciencia neoliberal. Tabula Rasa. 2008 [acesso em 2018 jun 2]; (9):247-283. Disponível em: https:// doi.org/10.25058/20112742.347.

31. Guimarães Neto L. Trajetória econômica de uma região periférica. Estud. Avanç. 1997 [acesso em 2019 set 2]; 11(29):37-54. Disponível em: https://doi.org/10.1590/ S0103-40141997000100003.

32. Rossi A. Women in Science: Why So Few?: Social and psychological influences restrict women's choice and pursuit of careers in science. Science. 1965 [acesso em 2019 ago 2]; 148(3674):1196-1202. Disponível em: https:// doi.org/10.1126/science.148.3674.1196.

33. Fox M. Women, Science, and Academia: Graduate Education and Careers. Gender and Society. 2001 [acesso em 2018 jun 2]; 15(5):654-666. Disponível em: https://doi.org/10.1177/089124301015005002.

34. Musselin C. Les universitaires. Paris: La Découverte; 2008. 
35. Probert B. 'I Just Couldn't Fit It In': Gender and Unequal Outcomes in Academic Careers. Gender, Work and Organization. 2005 [acesso em 2018 mar 2]; 12(1):50-72. Disponível em: https://doi.org/10.1111/ j.1468-0432.2005.00262.x.

36. Gupta N, Sharma A. Women Academic Scientists in India. Soc. Stud. Scienc. 2002 [acesso em 2018 mar 2]; 32(5-6):901-915. Disponível em: https://doi. org/10.1177/030631270203200505.

37. Ma Y, Zhao Y, Gong X, et al. Close the gender gap in Chinese science. Nature. 2018 [acesso em 2018 mar 2]; 557(7703):25-27. Disponível em: https://www.nature.com/articles/d41586-018-04996-3.

38. Assis C. Infográfico: Os caminhos de mulheres e homens na ciência brasileira - Gênero e Número. 26 jun 2018. [acesso em 2019 ago 2]. Disponível em: http:// www.generonumero.media/infografico-os-caminhos-de-mulheres-e-homens-na-ciencia-brasileira.

39. Olinto G. A inclusão das mulheres nas carreiras de ciência e tecnologia no Brasil. Inclusão Social. 2012 [acesso em 2020 ago 31]; 5(1). Disponível em: http:// revista.ibict.br/inclusao/article/view/1667.

40. Löwy I. Ciências e gênero. In: Hirata H, Laborie F, Le Doaré $\mathrm{H}$, et al. Dicionário crítico do feminismo. São Paulo: Ed. UNESP; 2009.

41. Weber J, Ramos C, Mester A, et al. Perfil dos pesquisadores bolsistas de produtividade científica em Psicologia do Conselho Nacional de Desenvolvimento Científico e Tecnológico. Estud. Psicol. 2015 [acesso em 2019 set 2]; 32(1):1-11. Disponível em: https://doi. org/10.1590/0103-166X2015000100001.

42. Negri F, Koeller P. O Declínio do investimento público em ciência e tecnologia: uma análise do orçamento do Ministério da Ciência, Tecnologia, Inovações e Comunicações até o primeiro semestre de 2019. Brasília, DF: IPEA; 2019.

43. Santos V. Uma "perspectiva parcial" sobre ser mulher, cientista e nordestina no Brasil. Rev. Estud. Fem. 2016 [acesso em 2019 nov 2]; 24(3):801-824. Disponível em: https://doi.org/10.1590/1806-9584-2016v24n3p801.

44. Staniscuaski F. Projeto: Parent in Science. In: $1^{\circ}$ Simpósio Brasileiro sobre Maternidade e Ciência; 10-11 maio 2018; Porto Alegre. Porto Alegre: PUCRS; 2018. [acesso em 2018 maio 30]. Disponível em: https:// www.parentinscience.com/.

45. Collins P. Pensamento Feminista Negro: conhecimento, consciência e a política do empoderamento. São Paulo: Boitempo; 2019.

46. Vieira B. Mulheres negras no Brasil: trabalho, família e lugares sociais. [dissertação]. Campinas: Faculdade de Educação da Universidade Estadual de Campinas; 2018.107 p.

47. Carvalhaes F, Ribeiro CAC. Estratificação horizontal da educação superior no Brasil: Desigualdades de classe, gênero e raça em um contexto de expansão educacional. Tempo soc. 2019 [acesso em 2019 dez 2]; 31(1):195-233. Disponível em: https://doi. org/10.11606/0103-2070.ts.2019.135035.

48. Mignolo W. Historias Locales/Diseños Globales: Colonialidad, Conocimientos Subalternos y Pensamiento Fronterizo. Madrid: Ediciones Akal; 2003.

49. Torres N. Sobre la colonialidad del ser: contribuciones al desarrollo de un concepto. In: Castro-Goméz S, Grosfoguel R, editores. El giro decolonial: Reflexiones para una diversidad epistémica más allá del capitalismo global. Bogotá: Pontificia Universidad Javeriana-Siglo del Hombre; 2007.

50. Carneiro S. A construção do outro como não-ser como fundamento do ser. [tese]. São Paulo: Universidade de São Paulo; 2005.

Recebido em 22/12/2020

Aprovado em 08/04/2021

Conflito de interesses: inexistente

Suporte financeiro: não houve 